\title{
COMPARISON OF GRADIENT-BASED EDGE DETECTORS APPLIED ON MAMMOGRAMS
}

\author{
CRISTIANA MOROZ-DUBENCO
}

\begin{abstract}
Breast cancer is one of the most common types of cancer amongst women, but it is also one of the most frequently cured cancers. Because of this, early detection is crucial, and this can be done through mammography screening. With the increasing need of an automated interpretation system, a lot of methods have been proposed so far and, regardless of the algorithms, they all share a step: pre-processing. That is, identifying the image orientation, detecting the breast and eliminating irrelevant parts.

This paper aims to describe, analyze, compare and evaluate six of the most commonly used edge detection operators: Sobel, Roberts Cross, Prewitt, Farid and Simoncelli, Scharr and Canny. We detail the algorithms, their implementations and the metrics used for evaluation and continue by comparing the operators both visually and numerically, finally concluding that Canny best suit our needs.
\end{abstract}

\section{INTRODUCTION}

According to Bray et. al [2], in 2018 breast cancer was the second leading type of cancer that caused death amongst women worldwide. It is also the most frequently diagnosed type of cancer when it comes to women, with a percentage of $25 \%$ of all types of cancer. Moreover, about $10 \%$ of women develop breast cancer [13].

Fortunately, early detection along with proper treatment can lead to curing. Mammography is one of the most used screening methods, which proved to be very effective and reliable in detecting cancer in early stages. Given the fact that it is recommended for mammographies to be performed at regular intervals and keeping in mind that mammogram interpretation is both difficult and time-consuming, the need for an automated interpretation system becomes imminent.

Received by the editors: 6 June 2021 .

2010 Mathematics Subject Classification. 68U10.

1998 CR Categories and Descriptors. I.4.6 [Image Processing and Computer Vision]: Segmentation - Edge and feature detection.

Key words and phrases. Image Processing, Computer Vision, Edge Detection. 
Although there are many different methods for mammography interpretation proposed in literature, most of them follow the same steps, as stated in Es-salhi et al. [6], Ramani et al. [18], Duque et al. [5], Sharma and Sharma [20] and Desai et al. [4], to name just a few:

(1) pre-processing: identification of the image orientation, detection of the breast, elimination of the background and detection and elimination of the pectoral muscle in case of medio-lateral orientation;

(2) segmentation: detection of possible lesion;

(3) classification: labeling the segmented area as either benign or malignant.

In this paper we are focusing on the first step and, more exactly, on breast detection. In order to perform an accurate segmentation, only the breast should be analyzed, whilst the black background and, especially, the artifacts, which are high-intensity areas that can affect the quality of the segmentation process, should be removed. In order to achieve this goal, we compared the performance of various existing edge detection operators when applied to mammograms - that is, we described each operator, analyzed their outputs, compared them both to the original images and to one another and applied a few well-known image quality assessment metrics on these results.

Whilst many comparative studies regarding the performance of popular edge detection techniques have been conducted so far, by the time we conducted this study we could not find any paper that compares the techniques when applied strictly to mammographic images which would either back the results by numerical values (namely, by computing image quality assessment metrics on the outputs) or apply those techniques on a large dataset.

In order to achieve these objectives, we applied existing edge detection methods on the mini-MIAS database [22].

\section{Scientific Problem}

Edge detection is a fundamental tool in image processing, used as a preprocessing step to feature detection, feature extraction and image segmentation. Usually, edges outline object boundaries, boundaries between objects and the background of the image, therefore allowing the extraction of the region of interest for further processing. In our case, we expect that the edge detection operation correctly and completely identifies the boundary between the breast and the background.

Mammographies are obtained by using a low-dose x-ray system and have some special features, which can lead to a rather difficult process of edge detection:

- they are grey-scale images;

- they can contain weak boundaries; 
- they exhibit Gaussian noise;

- they can contain a different type of background noise: artifacts, such as medical labels;

- they can have low quality, low contrast and poor illumination, depending on the machine used.

Edge detection aims to identify points at which the brightness is slightly different. These points are organized into a set of curved line segments, namely edges. Rosenfeld and Kak [19] defined edges as abrupt changes in gray level or texture at the intersection of two different regions, while Park and Murphey [16] differentiated between edge points and edge fragments, stating that edge points are pixels where the local intensity changes significantly and edge fragments represent the edge points along with their orientation. Therefore, edges are defined as pixels that have discontinuities in intensity. There are four different edge types:

(1) step edges: ideal type of edges that occur when the intensity changes significantly from one side to the other;

(2) line edges: edges that occur when the intensity changes significantly, remains at the new value for a number of pixels, then returns to the original value;

(3) ramp edges: step edges where the intensity does not change instantaneously, but the change occurs over a finite distance;

(4) roof edges: line edges where the intensity does not change instantaneously, but the change occurs over a finite distance.

Since edges are pixels that have abrupt changes in intensity, the derivatives of the image intensity function can be used to measure these discontinuities, either by thresholding the first derivative values or by searching for zero-crossings in the second derivative of the image function.

Edge detection techniques based on the derivatives of the image intensity functions are widely used and can be split into two categories: search based methods and zero-crossing based methods. Search based methods use a firstorder derivative expression in order to compute a measure of edge strength and then search for local directional maxima of the gradient magnitude, while zerocrossing based methods search for zero crossings in a second-order derivative expression.

Following, we are going to analyze several search based edge detection techniques. These methods, also called gradient-based methods, detect the edges by computing the gradient of local intensity at each point in the image and associate the local peaks in the first derivative with edges in the original image. 
Considering $f(x, y)$ an image with $(x, y)$ denoting the coordinates of a point, the two-dimensional gradient is a vector with two elements:

$$
G=\left[\begin{array}{l}
G_{x} \\
G_{y}
\end{array}\right]=\left[\begin{array}{l}
\frac{\partial f(x, y)}{\partial x} \\
\frac{\partial f(x, y)}{\partial y}
\end{array}\right]
$$

where $G_{x}$ and $G_{y}$ are measures for changes in pixel values in the horizontal and vertical directions, respectively.

Usually, gradient-based edge detection operators follow three steps:

(1) smoothing: pre-processing step for reducing the noise;

(2) differentiation: convolving the image with the two masks, $G_{x}$ and $G_{y}$, for computing the local gradient;

(3) detection: detecting edge points based on the local gradients.

In this paper, we are focusing on six well-known gradient-based edge detectors: Sobel [23], Prewitt [1], Roberts Cross [10], Scharr [21], Farid and Simoncelli [7] and Canny [3].

\section{Related Work}

In Ramani et al. [18], four filters, namely mean filter, median filter, adaptive median filter and wiener filter, are both visually and numerically compared on mammographies. The filters are applied on three images from the mini-MIAS database [22]. On each image, three different types of noise are applied: salt \& pepper noise, speckle noise and Gaussian noise. Afterwards, the images are reconstructed using the above-mentioned filters and the results are compared in terms of Mean Squared Error, Peak Signal to Noise Ratio, Structural Content and Normalized Absolute Error, concluding that the adaptive median filter yields the best results. Moreover, the numerical results are backed by the visual results obtained for one of the three images used for evaluation.

In paper [10], Maitra et al. propose a two-phase edge detection algorithm for breast detection in mammographies: homogenizing the image and detecting the edges. For the first phase, a novel method, which adjusts the intensity of the image in order to obtain a normalized intensity level, is presented. For the second phase, the input image is scanned in both horizontal and vertical direction and two edge maps are constructed, which are then merged to obtain the edge map of the image. The results obtained for five mammograms from the mini-MIAS database are visually compared to the results obtained with classical edge detection operators, namely Roberts Cross, Prewitt, Sobel, Kirsch and Laplacian of Gaussian, concluding that the proposed method produces the best results.

In Mirzaalian et al. [15], a new algorithm for breast contour detection is described, tested and compared to two other existing methodologies, presented by Ferrari et al. [8] and Wirth [25]. The proposed method is composed 
of multiple steps: normalizing the mammograms by histogram equalization, convolving with a mask, removing small noises through morphological operations, removing larger noises through labeling and convolving the top of the mammogram with a mask to overcome the problem of inaccurate border detection due to low contrast between the breast region and the background. When comparing the proposed method with the ones proposed by Ferrari et al. and Wirth for 20 mammograms in terms of Haudorff Distance Measure and Mean of Absolute Error Distance Measure, the proposed method outperformed the other two methods.

On the other hand, a number of comparative studies of edge detectors have been conducted so far. Harun et al. [9] compare five methods - Sobel, Canny, Roberts Cross, Canny and Sobel combination and Canny and Roberts Cross combination - in order to determine the vessel wall elasticity, by applying to operators on ten B-mode images and computing Mean Squared Error and Peak Signal to Noise Ratio on the output image, leading to the conclusion that the combination between the Canny and the Roberts Cross operators yields the most satisfactory results.

Kumar et al. [12] present a comparison of Prewitt, Sobel, Roberts Cross, Canny, Laplacian of Gaussian and Zero Crossing applied on three biometric images: the image of an iris, the image of a thumbprint and the image of a face. The numerical results are obtained by computing Mean Squared Error and Peak Signal to Noise Ratio, using, for each operator, the outputs of the other operators as ground truth. This paper concludes that Canny is the best fitted edge detection operator for biometric images.

Poobathy and Chezian [17] compare the performance of Canny, Sobel, Laplacian of Gaussian, Roberts Cross and Prewitt operators, by applying them on set of four universally standardized test images and computing Mean Squared Error and Peak Signal to Noise Ratio against the ground truth image. The authors achieve the conclusion that the Canny operator outperforms the other ones.

\section{Proposed Approach}

In order to compare the edge detection operators, we used a simple program that loads an image, pre-proccesses it and then applies the operator. For smoothing the image, we used the GaussianBlur method from the opencvpython library, while for edge detection, we used the scikit-image library in Python.

To evaluate the performances of the presented edge detection operators, we first visually compared their results, using three test images. For this goal, we applied each of the operators on the selected images and compared their 
outputs both to the original images and to one another. The resulting images are to be presented in the following section.

Secondly, we applied three quality assessment metrics on the entire dataset, as follows:

\section{(1) Mean Squared Error}

The Mean Squared Error (MSE) [12] is a measure for the average squared difference between the estimated values and the actual values - that is, the square difference between the compared images. It is a risk function which corresponds to the expected value of the squared error loss.

The mean squared error between two images can be expressed as:

$$
M S E=\frac{1}{M N} \sum_{x=1}^{M} \sum_{y=1}^{N}\left(I_{1}(x, y)-I_{2}(x, y)\right)^{2}
$$

where $M$ and $N$ are the number of rows and columns respectively in the input images (which need to be equal in order to obtain valid results) and $I_{1}(x, y)$ and $I_{2}(x, y)$ represent the value of the pixel having the coordinates $(x, y)$, in each image respectively.

The MSE is always equal or greater than zero, with better values being closer to zero.

(2) Peak Signal to Noise Ratio

The Peak Signal to Noise Ratio (PSNR) [11] indicates the level of losses or signals integrity. It is measured in decibels and is frequently used to measure the quality of compressed images in comparison to the original ones, with higher PSNR values representing a better quality of the modified image.

The peak signal to noise ratio is computed using the formula:

$$
P S N R=10 \log _{10}\left(\frac{R^{2}}{M S E}\right)
$$

where $R$ is the dynamic range of pixel values in the input image (which is 255 for gray level images where pixel values are represented as 8-bit integers) and $M S E$ is the mean squared error between the two images.

(3) Structural Similarity Index Measure

The Structural Similarity Index Measure (SSIM) [24] is more related to the human visual system, extracting information as contrast, structure and luminance. It aims to address the limitations of the MSE in terms of perceived similarity by taking texture into account. 
The SSIM index is computed on multiple windows of an image. For two windows $p$ and $q$ of size $S x S$, we have the following comparison functions, for luminance, contrast and structure:

$$
l(p, q)=\frac{2 \mu_{p} \mu_{q}+C_{1}}{\mu_{p}^{2}+\mu_{q}^{2}+C_{1}}, \quad c(p, q)=\frac{2 \sigma_{p} \sigma_{q}+C_{2}}{\sigma_{p}^{2}+\sigma_{q}^{2}+C_{2}}, \quad s(p, q)=\frac{\sigma_{p q}+C_{3}}{\sigma_{p} \sigma_{q}+C_{3}}
$$

where $\mu_{p}$ and $\mu_{q}$ are the averages of $p$ and $q$ respectively:

$$
\mu_{p}=\frac{1}{S} \sum_{i=1}^{N} p_{i} \quad \text { and } \quad \mu_{q}=\frac{1}{S} \sum_{i=1}^{N} q_{i}
$$

$\sigma_{p}$ and $\sigma_{q}$ are the variances of $p$ and $q$ respectively:

$$
\sigma_{p}=\left(\frac{1}{S-1} \sum_{i=1}^{S}\left(p_{i}-\mu_{p}\right)^{2}\right)^{\frac{1}{2}} \quad \text { and } \quad \sigma_{q}=\left(\frac{1}{S-1} \sum_{i=1}^{S}\left(q_{i}-\mu_{q}\right)^{2}\right)^{\frac{1}{2}}
$$

$\sigma_{p q}$ is the covariance of $p$ and $q$ :

$$
\sigma_{p q}=\frac{1}{S-1} \sum_{i=1}^{S}\left(p_{i}-\mu_{p}\right)\left(q_{i}-\mu_{q}\right)
$$

and $C_{1}, C_{2}$ and $C_{3}$ are constants, defined as follows:

$$
\begin{aligned}
& C_{1}=\left(K_{1} R\right)^{2} \text {, where } K_{1}<<1 \quad \text { is a small constant } \\
& C_{2}=\left(K_{2} R\right)^{2} \text {, where } K_{2}<<1 \quad \text { is a small constant } \\
& C_{3}=\frac{C_{2}}{2}
\end{aligned}
$$

By combining the three comparison functions presented above, we obtain the formula for the structural similarity index measure:

$$
\operatorname{SSIM}(p, q)=[l(p, q)]^{\alpha} \cdot[c(p, q)]^{\beta} \cdot[s(p, q)]^{\gamma}
$$

with $l, c$ and $s$ denoting the luminance, contrast and structure respectively, and $\alpha>0, \beta>0$ and $\gamma>0$ being the parameters used for adjusting the relative importance of the components. By setting $\alpha=\beta=\gamma=1$, we obtain a specific form of the index, which we are going to use in this paper:

$$
\operatorname{SSIM}(p, q)=\frac{\left(2 \mu_{p} \mu_{q}+C_{1}\right)\left(2 \sigma_{p q}+C_{2}\right)}{\left(\mu_{p}^{2}+\mu_{q}^{2}+C_{1}\right)\left(\sigma_{p}^{2}+\sigma_{q}^{2}+C_{2}\right)}
$$

In order to measure the quality of the output image from the edge detection operation as a whole, rather than the quality of particular windows of the image, we are going to use a mean structural 
similarity index measure:

$$
\operatorname{MMSIM}(P, Q)=\frac{1}{W} \sum_{i=1}^{M} \operatorname{SSIM}\left(p_{i}, q_{i}\right)
$$

where $P$ is the original image, $Q$ is the image obtained after applying an edge detection operator, $W$ is the number of windows in the image and $p_{i}$ and $q_{i}$ are the image contents at the i-th window.

Because of the fact that the dataset we used for experiments contains neither ground truth images for reference, nor any relevant information regarding the breast contour, we followed the methodology presented by Poobathy and Chezian [17], Mat Harun et. al [14] and Kumar et. al [12] and compared the results of the edge detection operators to the original image and to the outputs of the other operators. That is, we applied each metric on the output of each operator, first against the original image and afterwards against the outputs of the other operators. To get a numerical value for the entire dataset, we computed the average of the results of each individual metric for each image.

Normally, when comparing a processed image to the ground truth, MSE values closer to 0 indicate a better result. However, in our case, when using the original image as ground truth, as stated in Poobathy and Chezian [17], we are interested in higher values for MSE. MSE values closer to zero indicate an output very similar to the original image, which means that the operator was not able to properly detect the edges. Since we aim to determine which operator best detects the contour, we are looking for the output image that is the most different than the original one. Thus, higher values for MSE mean an output containing almost only the contour of the breast, with both the background and the inside of the breast being considered errors.

On the other hand, for PSNR we are looking for values closer to zero. Following the same logic as for MSE and taking into consideration the fact that the peak signal to noise ratio represents the measure of the peak error, the operators yielding lower results for PSNR better detect the contour.

Finally, we are using MSSIM to compute the similarity level between the outputs of the edge detectors and the original images. Because MSSIM is used to determine the structural similarity, we aim for values closer to zero, which would indicate that, from a structural point of view, the output is no longer similar to the original image.

When comparing the operators to one another, according to Kumar et. al [12], higher value of dissimilarities between one operator and the others indicate a better performing edge detection operator. In order to properly compare the operators, we consider the average value for each of the metrics for every operator and look for lower PSNR and MSSIM values and higher MSE values. 
For analyzing and comparing the performance of the edge detection operators, we use the mini-MIAS database [22], provided by the Mammographic Image Analysis Society, which contains 322 mammograms, digitized and reduced to 200 micro pixel edge, at a size of $1024 \times 1024$ pixels. The mammograms can be divided into three main categories: normal, containing benign abnormality and containing malignant abnormality. For visually comparing existing edge detection techniques we use three mammograms from the mini-MIAS database, one from each category, chosen randomly, where:

- mdb014 does not contain abnormalities;

- mdb080 contains a well-defined/circumscribed benign abnormality centered at $(432,149)$ coordinates with a radius of 20 pixels;

- mdb184 contains a spiculated malignant abnormality centered at $(352,624)$ coordinates with a radius of 114 pixels.

For numerical evaluation of the operators, we use all the images in the database.

\section{Experimental Results}

5.1. Visual Results. The results of applying the operators onto the original images are shown in Figure 1 as follows:

- the first column contains the original images,

- the second column contains the result obtained for the Sobel operator,

- the third column contains the result obtained for the Prewitt operator,

- the fourth contains the result obtained for the Roberts Cross operator,

- the fifth column contains the result obtained for the Scharr operator,

- the sixth column contains the result obtained for the Farid and Simoncelli operator,

- the seventh contains the result obtained for the Canny operator, with the threshold values chosen experimentally to $t_{\text {low }}=3$ and $t_{\text {high }}=10$.

For a better visualization of the results, we converted the edge detection results into binary images, where all the pixels detected as edges, regardless of their intensity, are shown in white, and the background is shown in black. The binary results are presented in Figure 2.

Given these visual comparisons, we consider that the best results were obtained using the Canny filter, although the Sobel and Roberts filters also yielded good results in terms of breast detection, but their results contain much more noise than Canny's. 


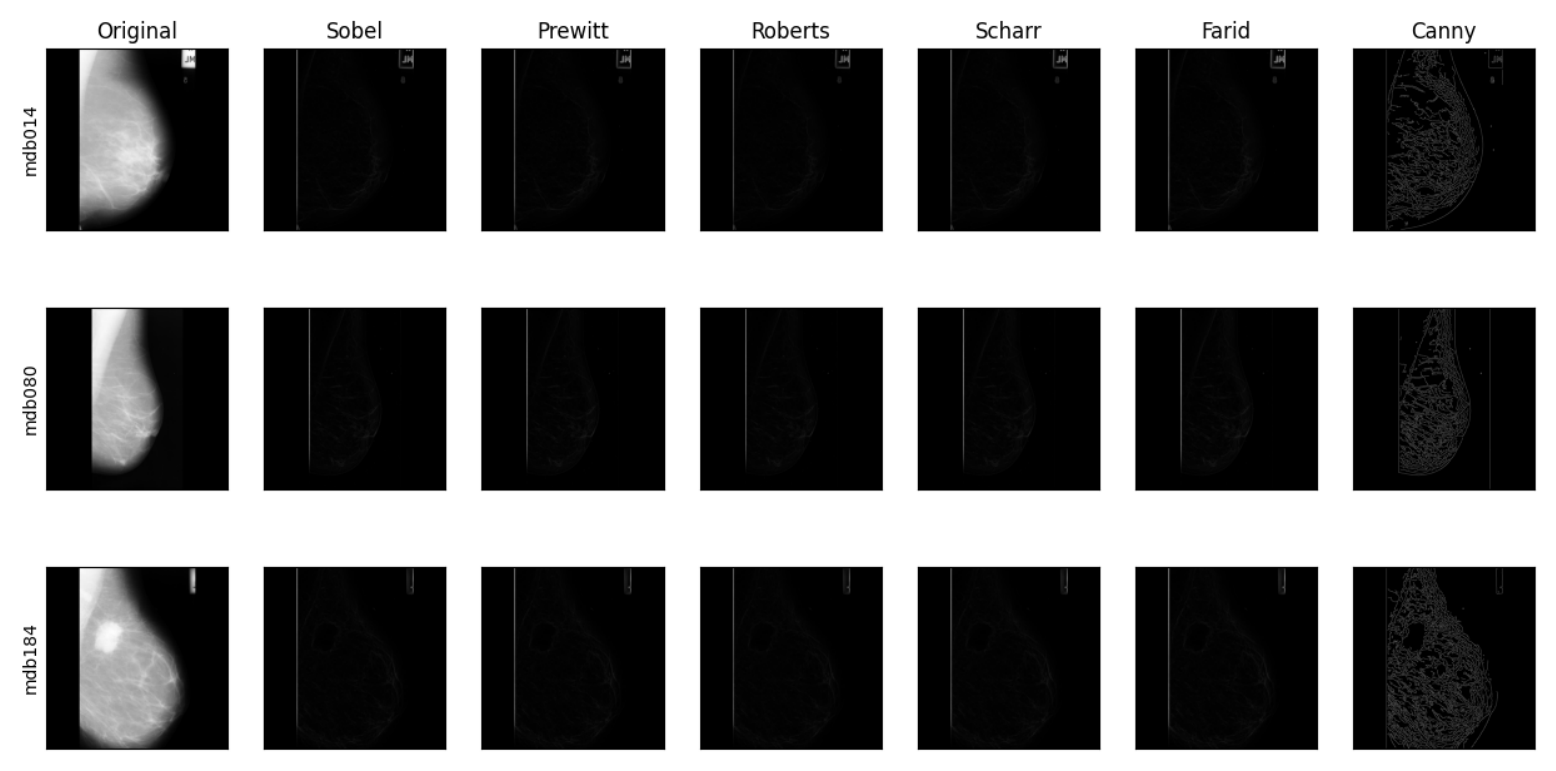

Figure 1. Comparison of edge detectors

5.2. Numerical Results. For comparing the operators numerically, we applied each operator on every image of the mini-MIAS dataset, then applied metrics as follows:

(1) Use the original image as ground truth and compute MSE, PSNM, MSSME for every image against the output image of every operator;

(2) Use each operator's output image as ground truth and compute MSE, PSNM, MSSME for every image against the output image of every operator.

The results presented in Table 1, Table 2, Table 3 and Table 4 represent the average of the results obtained across all 322 images in the mini-MIAS dataset.

Table 1 shows that the MSE values for all the operators are very close and, at the same time, very high. That means that the operators detected the edges and none of them produced an output close to the original image. The highest MSE value was obtained for the Canny operator, with a difference of approximately 0.6 from Farid, which produced the second highest value. The same table presents also the values obtained for PSNR, with Canny yielding 


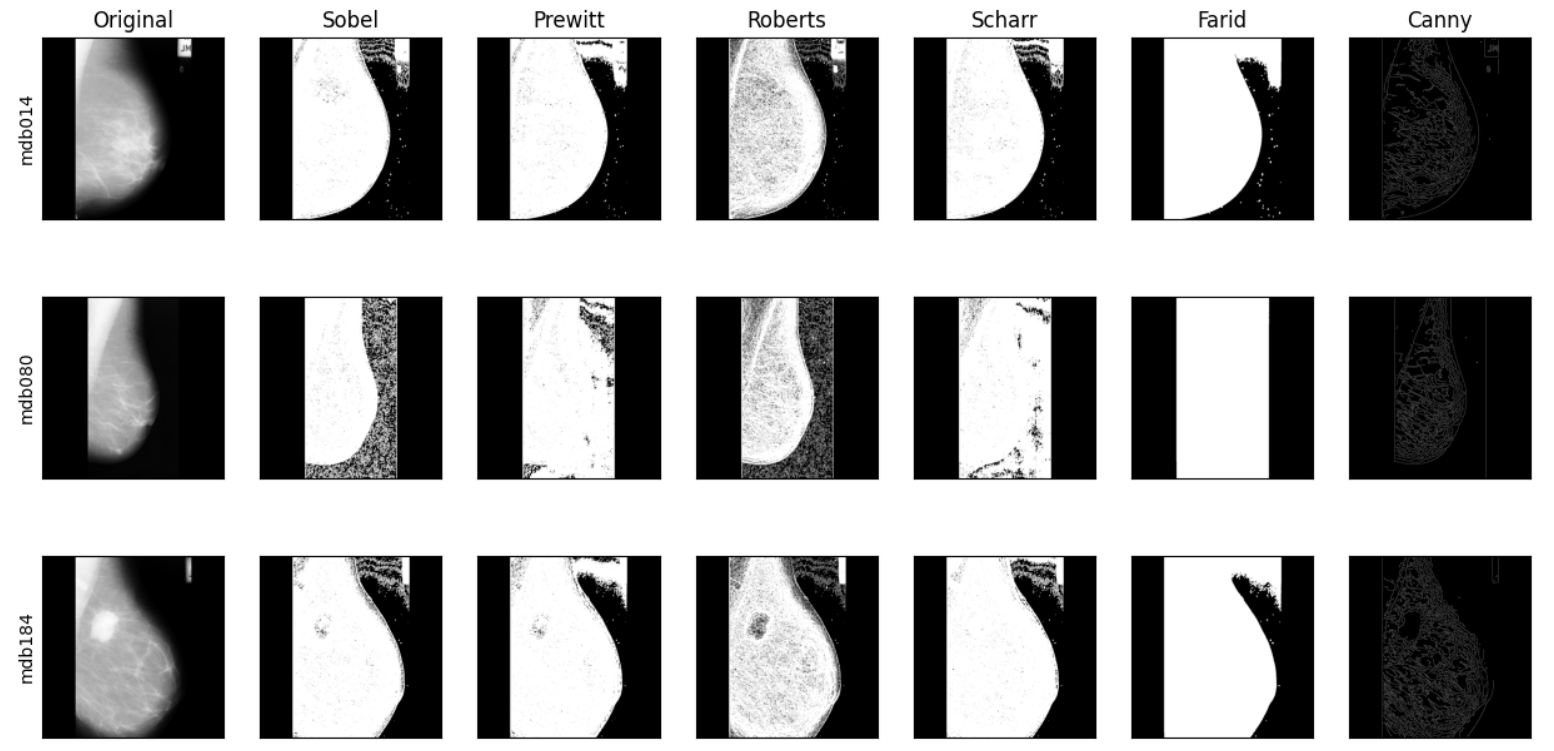

Figure 2. Comparison of edge detectors

\begin{tabular}{|c|c|c|c|}
\hline & MSE & PSNR & MSSMI \\
\hline Sobel & 8417.05056 & 9.17966 & 0.51232 \\
\hline Prewitt & 8417.07461 & 9.17965 & 0.51232 \\
\hline Roberts & 8417.16912 & 9.17960 & 0.512333 \\
\hline Scharr & 8417.02115 & 9.17968 & 0.51223 \\
\hline Farid & 8417.83910 & 9.17952 & 0.51223 \\
\hline Canny & 8418.43014 & 9.17893 & 0.51275 \\
\hline
\end{tabular}

TABLE 1. MSE, PSNR and MSSMI computed using the original image as ground truth

the lowest value, followed by Scharr, Sobel and Prewitt. As for MSSIM, all the operators produced similar values of around 0.5, meaning that the structure of the mammography has somehow changed. In this case as well, Canny raised the best result. 


\begin{tabular}{|c|c|c|c|c|c|c|}
\hline MSE & Sobel & Roberts & Prewitt & Farid & Scharr & Canny \\
\hline Sobel & 0 & $4.74 \mathrm{E}-05$ & $3.61 \mathrm{E}-08$ & 0.00010 & $2.08 \mathrm{E}-08$ & 0.03303 \\
\hline Roberts & $4.74 \mathrm{E}-05$ & 0 & $4.72 \mathrm{E}-05$ & $4.00 \mathrm{E}-05$ & $4.76 \mathrm{E}-05$ & 0.03338 \\
\hline Prewitt & $3.61 \mathrm{E}-08$ & $4.72 \mathrm{E}-05$ & 0 & 0.00010 & $1.11 \mathrm{E}-07$ & 0.03303 \\
\hline Farid & 0.00010 & $4.00 \mathrm{E}-05$ & 0.00010 & 0 & 0.00010 & 0.03373 \\
\hline Scharr & $2.08 \mathrm{E}-08$ & $4.76 \mathrm{E}-05$ & $1.11 \mathrm{E}-07$ & 0.00010 & 0 & 0.03302 \\
\hline Canny & 0.03303 & 0.03338 & 0.03303 & 0.03373 & 0.03302 & 0 \\
\hline
\end{tabular}

TABLE 2. MSE computed using the operators' outputs as ground truth

\begin{tabular}{|c|c|c|c|c|c|c|}
\hline PSNR & Sobel & Roberts & Prewitt & Farid & Scharr & Canny \\
\hline Sobel & inf & 43.64106 & 74.64643 & 40.22099 & 77.03130 & 15.01364 \\
\hline Roberts & 43.641068 & inf & 43.65951 & 44.36988 & 43.62168 & 14.96674 \\
\hline Prewitt & 74.64643 & 43.65951 & inf & 40.24118 & 69.74624 & 15.01292 \\
\hline Farid & 40.22099 & 44.36988 & 40.24118 & inf & 40.20274 & 14.92039 \\
\hline Scharr & 77.03130 & 43.62168 & 69.74624 & 40.20274 & inf & 15.01421 \\
\hline Canny & 15.01364 & 14.96674 & 15.01292 & 14.92039 & 15.01421 & inf \\
\hline
\end{tabular}

TABLE 3. PSNR computed using the operators' outputs as ground truth

\begin{tabular}{|c|c|c|c|c|c|c|}
\hline MSSIM & Sobel & Roberts & Prewitt & Farid & Scharr & Canny \\
\hline Sobel & 1 & 0.99341 & 0.99998 & 0.97973 & 0.99999 & 0.73985 \\
\hline Roberts & 0.99341 & 1 & 0.99355 & 0.99199 & 0.99328 & 0.74337 \\
\hline Prewitt & 0.99998 & 0.99355 & 1 & 0.98006 & 0.99996 & 0.73999 \\
\hline Farid & 0.97973 & 0.99199 & 0.98006 & 1 & 0.97943 & 0.74648 \\
\hline Scharr & 0.99999 & 0.99328 & 0.99996 & 0.97943 & 1 & 0.739703 \\
\hline Canny & 0.71237 & 0.72285 & 0.71283 & 0.73521 & 0.71190 & 1 \\
\hline
\end{tabular}

TABLE 4. MSSIM computed using the operators' outputs as ground truth

The results of computing MSE using each of the operator's outputs as ground truth are presented in Table 2. It is easily detectable that Canny produced the higher MSE values when computed using any other operator's output as reference. Also, by looking at the row for Canny in Table 3, one can tell that there are the lowest PSNR values. From table 4, we can tell that Sobel and Scharr produced almost identical results, while Canny's results are the most different from a structural point of view. 


\section{Conclusion And Future WOrk}

Suming up the results presented in the previous section, we can conclude that the Canny operator yields the best results. However, it is worth mentioning that the Farid and Simoncelli and the Scharr operators also yielded satisfactory numerical results, close to the ones obtained by Canny.

We consider that we reached our goal. We described, analyzed, compared and evaluated six edge detection operators - namely, Sobel, Roberts Cross, Prewitt, Farid and Simoncelli, Scharr and Canny -, providing useful visual and numerical comparison results.

As future work, we intend to compare the operators from a qualitative point of view as well, with the help of a radiologist, in order to back up the numerical results provided in this paper.

\section{Acknowledgments}

This work was supported by a grant of the Romanian Ministry of Education and Research, CCCDI - UEFISCDI, project number PN-III-P2-2.1-PED-20192607, within PNCDI III.

\section{REFERENCES}

[1] Ahmed, A. S. Comparative study among sobel, prewitt and canny edge detection operators used in image processing. J. Theor. Appl. Inf. Technol 96, 19 (2018), 65176525 .

[2] Bray, F., Ferlay, J., Soerjomataram, I., Siegel, R. L., Torre, L. A., And JEMAL, A. Global cancer statistics 2018: Globocan estimates of incidence and mortality worldwide for 36 cancers in 185 countries. CA: a cancer journal for clinicians 68,6 (2018), 394-424.

[3] CAnny, J. A computational approach to edge detection. IEEE Transactions on pattern analysis and machine intelligence, 6 (1986), 679-698.

[4] Desai, S. D., Megha, G., Avinash, B., Sudhanva, K., Rasiya, S., And LinGANAGOUDA, K. Detection of microcalcification in digital mammograms by improvedmmgw segmentation algorithm. In 2013 International Conference on Cloud \& Ubiquitous Computing \& Emerging Technologies (2013), IEEE, pp. 213-218.

[5] Duque, A. E. R., Gómez, D. C. A., and Nieto, J. K. A. Breast lesions detection in digital mammography: An automated pre-diagnosis. In 2014 XIX Symposium on Image, Signal Processing and Artificial Vision (2014), IEEE, pp. 1-5.

[6] Es-Salhi, R., Daoudi, I., Tallal, S., Medromi, H., et Al. A survey on segmentation techniques of mammogram images. In International Symposium on Ubiquitous Networking (2016), Springer, pp. 545-556.

[7] FARId, H., AND Simoncelli, E. P. Optimally rotation-equivariant directional derivative kernels. In International Conference on Computer Analysis of Images and Patterns (1997), Springer, pp. 207-214.

[8] Ferrari, R., Frere, A., Rangayyan, R., Desautels, J., and Borges, R. Identification of the breast boundary in mammograms using active contour models. Medical and Biological Engineering and Computing 42, 2 (2004), 201-208. 
[9] Harun, M., Izzah, M., Ibrahim, N., And Aziz, N. S. Comparative study of edge detection algorithm: vessel wall elasticity measurement for deep vein thrombosis diagnosis. ARPN Journal of Engineering and Applied Sciences 10, 19 (2015), 8635-8641.

[10] Indra Kanta Maitra, Sanjay Nag, S. K. B. A novel edge detection algorithm for digital mammogram. International Journal of Information and Communication Technology Research (2012).

[11] Jose, A., Dixon, K. D. M., Joseph, N., George, E. S., And Anjitha, V. Performance study of edge detection operators. In 2014 International Conference on Embedded Systems (ICES) (2014), IEEE, pp. 7-11.

[12] Kumar, S., Singh, M., AND Shaw, D. Comparative analysis of various edge detection techniques in biometric application. International Journal of Engineering and Technology (IJET) 8, 6 (2016), 2452-2459.

[13] LAU, T.-K., AND Bischof, W. F. Automated detection of breast tumors using the asymmetry approach. Computers and biomedical research 24, 3 (1991), 273-295.

[14] Mat Harun, N. H., Ibrahim, N., And Aziz, N. S. Comparative study of edge detection algorithm: vessel wall elasticity measurement for deep vein thrombosis diagnosis.

[15] Mirzadian, H., Ahmadzadeh, M. R., Sadri, S., and Jafari, M. Pre-processing algorithms on digital mammograms. In MVA (2007), pp. 118-121.

[16] Park, J., And Murphey, Y. Edge Detection in Grayscale, Color, and Range Images. 042008.

[17] Poobathy, D., and Chezian, R. M. Edge detection operators: Peak signal to noise ratio based comparison. IJ Image, Graphics and Signal Processing 10 (2014), 55-61.

[18] Ramani, R., Vanitha, N. S., and Valarmathy, S. The pre-processing techniques for breast cancer detection in mammography images. International Journal of Image, Graphics and Signal Processing 5, 5 (2013), 47.

[19] Rosenfeld, A., And KaK, A. Digital picture processing academic press. New York (1982), 242.

[20] Sharma, J., And Sharma, S. Mammogram image segmentation using watershed. Int $J$ Info Tech and Knowledge Management 4 (2011), 423-5.

[21] Sian, C., JiYe, W., Ru, Z., And Lizhi, Z. Cattle identification using muzzle print images based on feature fusion. In IOP Conference Series: Materials Science and Engineering (2020), vol. 853, IOP Publishing, p. 012051.

[22] SUCKLING J, P. The mammographic image analysis society digital mammogram database. Digital Mammo (1994), 375-386.

[23] Vairalkar, M. K., AND Nimbhorkar, S. Edge detection of images using sobel operator. International Journal of Emerging Technology and Advanced Engineering 2, 1 (2012), 291-293.

[24] Wang, Z., Bovik, A. C., Sheikh, H. R., And Simoncelli, E. P. Image quality assessment: from error visibility to structural similarity. IEEE transactions on image processing 13, 4 (2004), 600-612.

[25] WiRTh, M. A. A nonrigid approach to medical image registration: matching images of the breast. Royal Melbourne Institute of Technology, 1999.

Department of Computer Science, Faculty of Mathematics and Computer Science, Babeş-Bolyai University, Mihail Kogălniceanu 1, 400084, Cluj-Napoca, RoMANIA

Email address: cristiana.moroz@ubbcluj.ro 